\title{
Identification of an Active Component in Guiera senegalensis Plant Used for Healing Diabetes Wounds
}

\author{
Mohammed Sulieman Ali Eltoum*, Ahmed Hapeepallah Adam, Hassan Alshafee Mohamed, \\ Shomw Morwan Abody
}

Scientific Laboratories (Chemistry), Sudan University of Science and Technology, Khartoum, Sudan

Email address:

abotrtee174@gmail.com (M. S. A. Eltoum)

${ }^{*}$ Corresponding author

\section{To cite this article:}

Mohammed Sulieman Ali Eltoum, Ahmed Hapeepallah Adam, Hassan Alshafee Mohamed, Shomw Morwan Abody. Identification of an Active Component in Guiera Senegalensis Plant Used for Healing Diabetes Wounds. International Journal of Pharmacy and Chemistry. Vol. 6, No. 1, 2020, pp. 6-10. doi: 10.11648/j.ijpc.20200601.12

Received: March 3, 2020; Accepted: March 24, 2020; Published: May 12, 2020

\begin{abstract}
The African species Ghobash (Guiera senegalensis J. F. Gmel), is a small herb found mainly in West Africa. It had wide spread used in traditional medicine. The aim of this study is to identify the bioactive ingredient responsible of healing diabetes wound. The aim was obtained using different process; first the plant crude oil was extracted using the soxhlet apparatus and two organic solvents (Chloroform and Methanol). Then the natural products present in dry plant leave were investigated by phytochemical screening analysis tests which confirm the main classes of secondary metabolites, namely terpenoids, alkaloids, flavonoids and steroides. The anti-bacterial activity test was carried out to show the bioactivities properties of the plant. The tests were positive in two types of bacteria: staphylococcus aureus and pseudomonas aeruginosa. The (GC/MS) analysis was carried out to separate and identify the components of the plant and their structures. The obtained results showed the presence of different active ingredients such as: flavonoids, alkaloids and steroids in high percentage. The expected compound responsible of healing diabetes wound was suggested to be stigmasterol.
\end{abstract}

Keywords: Guiera Senegalensis, J. F Gmel, Active Component, Healing Diabetes Wounds, Stigmasterol

\section{Introduction}

Guierasenegalensis is one of the most popular West African medicinal plants, and is used to treat a wide variety of diseases. Its uses are comparable to those of CombretummicranthumG. Don, commonly called 'kinkeliba', and the plants are often used combined, especially, to treat common colds, fever and respiratory problems. The bitter leaves are most frequently used in Sudan aleaf is taken to treat diabetes. The wood is used for the framework of wells, bed posts and roof lattice work and it is also, commonly, used to fence farms. It is also an important source of fuel. The roots are split and used as chew sticks and tooth picks. The fruit yields a black dye. The leaves enter into various medico-magical preparations, e.g. to free people from evil spirits and to bring good luck. Leaves and roots contain $\beta$ carboline alkaloids $(0.15-0.2 \%), \quad$ and the methoxylatednaphthyl butanone guieranone A. In the roots tetrahydroharman (eleagnine) was the main alkaloid, with harman and harmalan (dihydroharman) as minor compounds. The leaves contain the alkaloids tetrahydroharman and harman, as well as guieranone $\mathrm{A}$.

Tannins were present in large amounts in all plant parts, including the galls. The different plant parts showed quantitative and qualitative differences with respect to the chemical composition of the tannins. Nine gallotannins with a quinic acid core and two condensed tannins (epicatechin and epigallocatechin gallate) have been isolated so far. The major tannin in all plant parts is 3,4,5-tri-O-galloylquinic acid. From the leaves, roots and galls a range of flavonoids was isolated, including catechin, myricitrin, several myricetin derivatives, rutin, rhamnetin, quercetin, 
quercetrin, kaempferol, tiliroside, apigenin and gallic acid. From the leaves the naphthopyrones 5methyldihydroflavasperone and 5-methylflavasperone and the amino acid ascorbic acid have been isolated. Mucilage was also present in all plant parts, but mostly in the fruits. Guiera senegalensis is acclaimed as a common herbal antipyretic and antimalarial among some tribal groups in northern Nigeria. Leaf extracts of the plant were thus tested for antiplasmodial, analgesic and anti-inflammatory effects in vivo. Results indicated the safe dose of extracts as 600 $\mathrm{mg} / \mathrm{kg}$ body weight of mice with $\mathrm{LD}_{50}$ of $1100 \mathrm{mg} / \mathrm{kg}$ bw. Only the methanolic fraction had antiplasmodial effect while ethylacetate and hexane fractions were ineffective. [3] Furthermore the methanolic extract produced a significant $(p<0.05)$ suppression of up to $67.52 \%$ levels. The extracts had no prophylactic effect and high parasitaemia including mortality of sub-inoculated mice were obtained on day 14 post treatment. [3] It gave $44.83 \%$ analgesic effect but was devoid of anti-inflammatory activity. Phytochemical screening indicated the presence of alkaloids, glycosides, tannins and flavonoids [3]. The antimicrobial activities of aqueous and methanol extracts of Guiera senegalensis were studied against some enteropathogens by agar-well diffusion method. Both extracts inhibited the growth of Streptococcus Pneumonia, Escherichia coli, Staphylococcus aureus, Enterobacter cloacae, Salmonella spp and Pseudomonas euroginosa. [3] The methanol extract was more effective on S. Pneumonia, E. coli, S. aureus, E. cloacae, Salmonella spp, while the aqueous extract was more active on Salmonella spp. and P. euroginosa. [3] The methanol extract showed quicker curative action in, experimentally, induced diarrhoea in adult male mice when administered once or twice at a dose of $556 \mathrm{mg} / \mathrm{kg}$ daily. [4] From this work, $G$. senegalensis root appears to be an effective measure for treating diarrhoea. Where foodpoisoning or metal poisoning are the causative agents. The phytochemical screening results of the aqueous extract, showed the presence of tannins, saponins, alkaloids, cardiac glycosides, coumarineand anthraquinones.

However [4] anthraquinones were not detected in the methanol extract and cardiac glycosides where not detected in the aqueous extract. Ethno medicinal use of this plant in the treatmentof diarrhoea is justified by this work and is encouraged [4]. Recent study the claimed antimicrobial activity of Sudanese medical plant, Guierasenegalensis leaves studies. This involved preparation and use of metanolic extract of Guierasenegalensis leaves. The study conducted on Guinea pig and rate with induced inhibition and slight contraction. The extract showed dose dependant inhibition on force and rate f contraction of isolated Guinea pig atrium. In isolated rate uterus preparation the extract showed slight contraction which blocked by $(2 \mathrm{mg} / \mathrm{mL}-1)$ cyproheptidine [5]. The chemical composition of total alkaloids from leaves and roots of Guiera senegalensis was investigated [6]. Three beta-carboline alkaloids were purified: in addition to harman and tetrahydroharman, known in roots and leaves, harmalan (dihydroharman) was isolated for the first time from roots of Guiera senegalensis. Guieranone A, a naphthylbutenone, was also purified from leaves and roots. The in vitro antiplasmodialactivity and the cytotoxicity of extracts and pure compounds were evaluated. Each total alkaloid extract and beta-carboline alkaloids presented an interesting antiplasmodial activity associated with a low cytotoxicity. Harmalan was less active than harman and tetrahydroharman. Guieranone A showed a strong antiplasmodial activity associated with a high cytotoxicity toward human monocytes. Its cytotoxicity was performed against two cancer cell lines and normal skin fibroblasts in order to study its anticancer potential: guieranone A presented a strong cytotoxicity against each cell strains. Finally, evaluated the potent synergistic antimalarial interaction of Guiera senegalensis and two plants, commonly, used in traditional remedies: Mitragynainermis and Pavetta crassipes. Three associations evaluated were additive. A synergistic effect was shown between total alkaloids extracted from leaves of Guierasenegalensis and those of Mitragynainermis. This result justified the traditional use of the plants in combination to treat malaria.

GC-MS analysis and antifungal activity from galls of Guiera senegalensis J. F Gmel (Combretaceae) have been investigated [7] The $G$ senegalensis exhibited an interesting antifungal activity against all strains tested. Quantitative phytochemical and elemental analysis of Guiera senegalensis leaf extract was carried out [8] the values of all the elements analyzed compares favorably with values obtained for other plants and thus indicated that G. senegalensis leaf contain significant amount of essential mineral elements. Their quantity is in the order $\mathrm{Ca}>\mathrm{K}>\mathrm{P}>\mathrm{Na}>\mathrm{Mg}>\mathrm{Fe}>\mathrm{Zn}>\mathrm{Cu}$. General Phytochemical Screening and Antioxidant Activity of Some Sudanese Medicinal Plants including guiera senegalesis were reported [9] and Acaricidal properties of two extracts from Guiera senegalensis J. F. Gmel. (Combrataceae) against Hyalomma anatolicum (Acari: Ixodidae) was studied [10]. Antitoxic, Antifungal and Phytochemical Analysis of Medicinal Compounds of Guiera senegalensis had been extensively studied by many authors [11-13]. Effects of extraction solvents on total phenolic and flavonoid contents and biological activities of extracts was also investigated [14], recently. Phytochemical Analysis, Cytotoxcity and Antifungal Activities of Guiera Senegalensis Leaves Extract Review was carried out [15] The aim of the review was to investigate the phytochemical analysis, toxicity, and the antifungal activity of Gueira senegalensis leaves extract and compare methods and they concluded that more research is needed to investigate if there is any side effect when the extract is taken orally. Further, the medicinal properties of the phytochemical compounds of Gueira senegalensis need to be further investigation. The aim of this study isto Identify of an Active Component in Guiera senegalensis Plant Used for Healing Diabetes Wounds. 


\section{Materials and Methods}

\subsection{Materials}

i Samples of Guierasenegalensis leaves were collected from West Sudan, Northern Kordfan (700Km west khartoum)

ii All chemicals used were of analytical grade type and includes:

Chloroform (99\%), Methanol (99\%), Wagner's reagent, Hager's reagent, Alkaline reagent, Copper acetate, Hydrochloric acid, Lead acetate, deionized Water.

iii Micro Organism (Staphylococcus aureus ATCC6538 Passage No 4, Pseudomonas aeruginosa ATCC9027 Passage No 4).

iv Glass ware and Apparatus:

Soxhlet. Apparatus, Beakers, Flasks, Petri dishes, pippete v Instrument:

Gas Chromatography Mass Spectrometry (GC/MS).

Instrument information:

Detector: Mass spectrometer

Model: GC/MS-QP2010Ultra

Company: Shimadzu

Country: Japan

Column: Rtx-5MS... Length (30)... Diameter $(0.25) \ldots$

Thickness $(0.25 \mu \mathrm{I})$

Carrier gas: Helium

\subsection{Methods}

Sample preparation for photochemical screening:

Guiera senegalensis leaves were collected from West Sudan, Northern Kordfan (700Km west khartoum) and dried in shadow. $10 \mathrm{~g}$ of the dried leave were ground by mortar and extracted by soxhlet using chloroform, Extraction was repeated using methanol.

Phytochemical screen:

Alkaloid test:

a) In dry and clean test tube $1 \mathrm{ml}$ of the extract were added and 3 drops of dilute Hydrochloric acid then it were treated with Wagner's reagent (Iodine in Potassium Iodide). the formation of brown /reddish precipitate indicates the presence of alkaloids.

b) $1 \mathrm{ml}$ of the extract were added to dry and clean test tube and 3 drops of dilute Hydrochloric acid were added then it were treated with Hager's reagent (saturated Picric acid solution). the presence of alkaloids confirmed by the formation of yellow colored precipitate.

Phytosterols, libermann test, $2 \mathrm{ml}$ of the extract few drops of chloroform, 3 drops of acetic anhydride, and 1 drop of concentrated sulphuric acid were added. The appearance of purple color shows the presence of steroid.

Terpens test, Copper actate test, The extract were dissolved in water and treated with 3 drops of copper acetate solution. Formation of emerald green colour indicates the presence of diterpens.

Flavonoids:

a) Alkaline Reagent test: In clean and dry test tube $1 \mathrm{ml}$, of extract, was treated with few drops of sodium hydroxide solution. Formation of intense yellow color which becomes colorless on addition of dilute acid, indicates the presence of flavonoids.

b) Lead Acetate test: In dry and clean test tube $1 \mathrm{ml}$, of extract was treated with, few, drops of lead acetate solution. Formation of, ayellow, precipitate indicates the presence of flavonoids.

Anti-Bacterial test:

Preparation of Chloroform extract (Reagent 1):

$0.5 \mathrm{~g}$ of chloroform extracted sample were dissolved in 2.5 $\mathrm{ml}$ of Methanol (Absolute) analytical grade, well, mixed and sonicated for $15 \mathrm{~min}$, the volume was completed to $20 \mathrm{ml}$ with water for injections, well mixed and used.

Preparation of Methanol extract (Reagent 2):

$0.396 \mathrm{~g}$ of methanol extracted sample were dissolved in $2.5 \mathrm{ml}$ of absolute Methanol analytical grade, mixed well then sonicated for $15 \mathrm{~min}$, the volume completed to $20 \mathrm{ml}$ with water, for injections, well mixed, and used.

\subsection{Methodology of Antimicrobial Tests}

In a rack of 20 test tubes $1 \mathrm{ml}$ of tryptone soya broth placed in each tube, tltted in screw caped bottle. At $121^{\circ} \mathrm{C}$ for $15 \mathrm{~min}$ the media were sterilized using an autoclave, at $121^{\circ} \mathrm{C}$ and 15 bar pressure, and left Then to cool at room temperature.

Suspensions of organisms were prepared in normal saline and compared with Macfarland turbidity. Reagents (1\&2) were diluted in four, separate, set, of ten test tubes, with previously prepared tryptone soya broth (each reagent in 2 set of tubes), suspensions of $1 \mathrm{ml}$ of organisms were added in four set of tubes (each bacterium suspension in two set of different diluted Reagent). After mixing the four set were incubated at $32^{\circ} \mathrm{C}$ for 24 hour.

\section{Results}

Tables 1 and 2 showed the results of Phytochemical screening of the Chloroform and Methanol extract respectively whereas table 3 showed the Anti-bacterial activity of the extracts.

Table 1. Resultsof phytochemical screeing of the Chloroform extract.

\begin{tabular}{lll}
\hline Natural Product Test & Name of Reagent & Results \\
\hline \multirow{2}{*}{ Alkaloids } & Wagner, s & + \\
& Hagers & + \\
Flavonoids & Alkaline & + \\
Terpens & Lead Acetate & + \\
Phytostrols & Copper Acetate & + \\
\hline
\end{tabular}


Table 2. Phytochemical screening of the Methanol extract.

\begin{tabular}{lll}
\hline Natural Product Test & Name of Reagent & Results \\
\hline \multirow{2}{*}{ Alkaloids } & Wagner, s & + \\
& Hagers & + \\
Flavonoids & Alkaline & + \\
Terpens & Lead Acetate & + \\
Phytostrols & Copper Acetate & + \\
\hline
\end{tabular}

Table 3. Anti-bacterial activity of the extracts.

\begin{tabular}{lll}
\hline Bacteria Organism & Type of extract & \%Minimum Inhibitory concentration (MIC) \\
\hline \multirow{2}{*}{ Staphylococcus aureus ATCC6538 Passage No 4 } & Chloroform & $1.25 \%$ \\
Pseudomonas aeruginosa ATCC9027 Passage No & Chloroform & $2 \%$ \\
4 & Methanol & $2.5 \%$ \\
\hline
\end{tabular}

Table 4. Peak report of TIC.

\begin{tabular}{lllll}
\hline Peak & R. Time & Area & Area $\%$ & Name \\
\hline 6 & 22.049 & 803094 & 2.87 & Stigmasterol \\
\hline
\end{tabular}
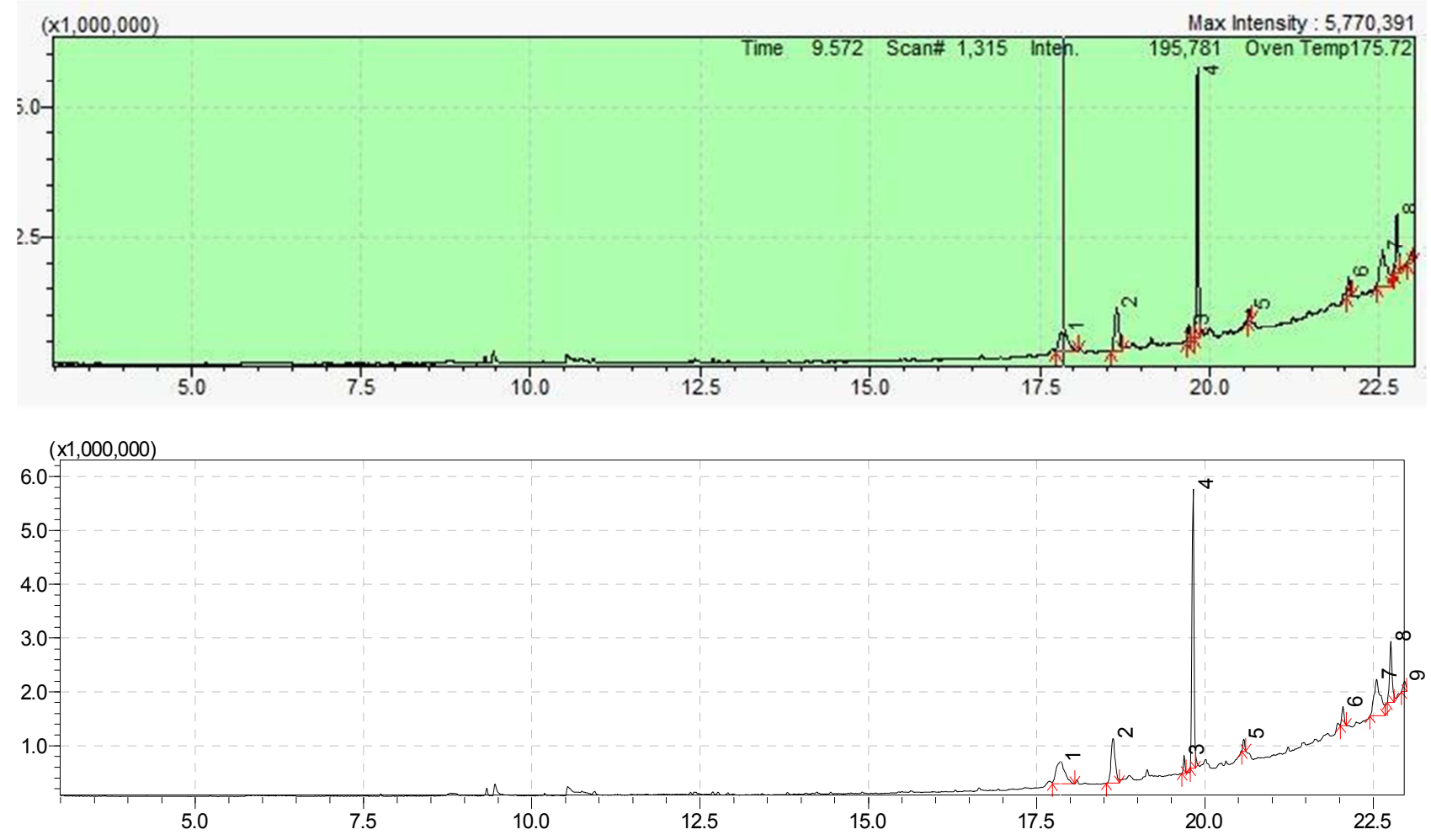

Figure 1. Gas chromatography mass spectrometry chromatogram of the mixture extract of the GuieraSeneglensis dry leaves.

\section{Discussion}

The phytochemical screening shows the presence of natural products as the results show in Tables 1 and 2 Identical quantities of alkaloids, terpens, flavonoids and steroids were detected in the two solvents extracts which explain the high value of the use of this plant in the traditional medicine.

Antibacterial test, show significant activity against two types of bacteria, gram-positive coccal bacterium and gramnegative, rod-shaped bacterium which classify this plant as one of strong antibiotics that work in wide spectrum as shown in Table 3.

GC/MS analysis show many bioactive components as shown in Tables 4 and Figure 1 which lead to identification of the component used of healing diabetes wound, the main objective of this study. This component is Stigmasterol which industrial company uses as the starting raw material for the synthesis in medical field which reduces inflammations, swellings and attendant pain and at the site of injury.

One of the components of the (GC/MS) analysis is Quiniacid which is aquinine derivative used to treat malaria. This explain the antiplasmodium activity of plant extract. 
These components have different activities which explain the many uses of this plant in traditional medicine.

\section{Conclusion}

In the present study the Guiera senegalensis J. F. Gmel species extract were investigated, the phytochemical screening showed the presence of alkaloid, terpens, flavonoids and steroids, the GC/MS analysis show different bioactive components which confirmed by the activity against some microorganism.

The bioactive component responsible for healing diabetes wound was identified as Stigmasterol.

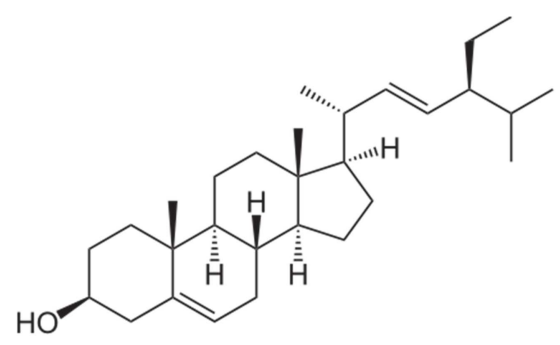

Figure 2. Stigmasterol.

\section{Recommendations}

The use of traditional, herbal, medicine is recommended for the following reasons:

1. More available.

2. Less expensive

3. limited risk of contamination or expiry.

4. No side effects as general.

\section{References}

[1] Chemistry for Pharmacy Students General, Organic and Natural Product Chemistry, Satyajit D. Sarker, University of Ulster, Coleraine, Northern Ireland, UK, Lutfun Nahar University of Ulster, Coleraine, Northern Ireland, UK [283 288].

[2] Plant Resources of Tropical Africa (PROTA) http://uses.plantnetproject.org/en/Guiera_senegalensis_\%28PROTA\%29.

[3] A. Jigam, O. Helmina, Akanya, E. N. Dauda and O. Emmanuel Ogbadoyi (2011). Antiplasmodial, analgesic and anti-inflammatory effects of crude Guiera senegalensis Gmel (Combretaceae) leaf extracts in mice infected with Plasmodium berghei. Journal of Pharmacognosy and Phytotherapy, 3 (10): 150-154.

[4] E. T. Williams, J. T Barminas, J. Akinniyi, and A. William (2009). Antidiarrhoeal effects of the root extracts of Guiera senegalensis in male mice. African Journal of Pure and Applied Chemistry, 3 (8): 152-157.

[5] A. E. A. Baragob, Abdelwahab Hassan, Maram Mohammed Algili Ismail, H. A. Samia, S. M. E. Khojali, A. O. A. Zakia, W. H. Almalki and H. E. H. FaragAlla (2013) Study of antimicrobial activities of Guiera senegalensis leaves. Research Journal of Pharmacology, 7 (3): 29-36.

[6] J. Fiot, S. Sanon, N. Azas, V. Mahiou, O. Jansen, L. Angenot, G. Balansard, E. Oliver. (2006). Phytochemical and pharmacological study of roots and leaves of Guiera (Combretaceae). J Ethnopharmacol, 106 (2): 173-8.

[7] P. A. Sombié, K. Konate, E. Youl, A. Y Coulibaly, M. Kiendrébéogo, M. I. Choudhary, O. G. Nacoulma (2013). GCMS analysis and antifungal activity from galls of Guiera senegalensis J. F Gmel (Combretaceae). Journal of Applied Pharmaceutical Science, 3 (12): 006-012.

[8] S. Y. Mohammed (2013). Quantitative phytochemical and elemental analysis of Guiera senegalensis leaf extract. Journal of Pharmacognosy and Phytotherapy, 5 (12): 204-207.

[9] M. E. Osman, H. H. Yassen, J. D. Deng, R. O. Mustafa, S. M. Hussein (2014). General Phytochemical Screening and Antioxidant Activity of Some Sudanese Medicinal Plants including guiera senegalesis. JOURNAL OF FOREST PRODUCTS \& INDUSTRIES, 3 (6): 292-295.

[10] I. M. Osman, A. S. Mohammed, A. B. Abdalla (2014). Acaricidal properties of two extracts from Guiera senegalensis J. F. Gmel. (Combrataceae) against Hyalomma anatolicum (Acari: Ixodidae). journal of Veterinary Parasitology, 199 (34): 201-205.

[11] N. K. Al Shafei, A. E. Elsha, and A. Nour (2016). Antitoxic, Antifungal and Phytochemical Analysis of Medicinal Compounds of Guiera senegalensis Leaves in Sudan. J Plant Biochem Physiol, 4: 2.

[12] A. Adebiyi, R. Gbekele-Oluwa Ayo, J. D. Habila (2016). Phytochemical Screening and Anti-Tb Activity of Root Extracts of Guiera senegalensis (J. F. Gmel). Biology, DOI: 10.11648/j.bio.20150306.20 • Corpus ID: 53054972.

[13] Z. M. Anka, Vijender Singh, S. N. Gimba, Gunjan Singh (2020). Antitoxic, Antifungal and Phytochemical Analysis of Medicinal Compounds of Guiera senegalensis Leaves. journal of drug Delivery \&Therapeutics, 10 (2).

[14] A. I. Dirar a, b, D. H. M. Alsaadi a, M. Wada a, M. A. Mohamed c, T. Watanabe a, H. P. Devkota (2019). Effects of extraction solvents on total phenolic and flavonoid contents and biological activities of extracts from Sudanese medicinal plants. South African Journal of Botany, 120: 261-267.

[15] H. M. Gurama, F. M. Maude, M. U. Jibrin, O. S. Oluwatovi, A. A. Sani, M. A. Inuwa, S. Yahaya and U. P. Chikere (2020). Phytochemical Analysis, Cytotoxcity and Antifungal Activities of Guiera Senegalensis Leaves Extract Review. Chemical \& Pharmaceutical Research, 2 (1): 1-4. 